CORRIGENDUM

\title{
Cooperation of signalling pathways in embryonic mammary gland development
}

\section{Gertraud W. Robinson}

Nature Reviews Genetics 8, 963-972 (2007)

In the last two paragraphs of the 'Ductal outgrowth' section on page 970 of this Review, the name of the p190-B RhoGAP gene was incorrectly referred to as RASGRF1. The corrected paragraphs are shown below.

The author apologizes for the error.

p190-B RhoGAP and IGF1 stimulate bud growth. A function in mammary mesenchyme induction and ductal growth has also been found for p190-B RhoGAP (encoded by Arhgap5), a negative regulator of the Rho pathway ${ }^{39}$. A role for p190-B RhoGAP in mammary biology was first postulated when the gene that encodes it was identified as being highly upregulated in terminal end buds, the structures that show highest proliferative activity in the ductal tree at puberty ${ }^{40}$. p190-B RhoGAPdeficient embryos have much smaller epithelial buds at E14.5 (REF. 39). The epithelial cells are disorganized and the cells in the mammary mesenchyme lack expression of steroid receptors.

Absence of insulin receptor substrate 1 (IRS1) and IRS2, which are downstream mediators of the insulin-like growth factor 1 receptor (IGF1R), results in the same defects as seen in p190-B RhoGAP-deficient embryos. This suggests that insulin-like growth factor 1 (IGF1) signalling through p190-B RhoGAP is important for the formation of a normal-sized mammary bud and the induction of mammary mesenchyme cell identity. 